\title{
Benefit-risk of Patients' Online Access to their Medical Records: Consensus Exercise of an International Expert Group
}

\section{Primary Health Care Informatics Working Group Contribution to the Yearbook of Medical Informatics 2018}

\author{
Harshana Liyanage', Siaw-Teng Liaw², Emmanouela Konstantara', Freda Mold ${ }^{3}$, Richard \\ Schreiber ${ }^{4}$, Craig Kuziemsky ${ }^{5}$, Amanda L. Terry ${ }^{6}$, Simon de Lusignan' ${ }^{1}$ \\ Department of Clinical \& Experimental Medicine, University of Surrey, GUILDFORD, Surrey, UK \\ 2 School of Public Health \& Community Medicine, UNSW Medicine Australia, Ingham Institute of \\ Applied Medical Research, NSW, Australia \\ 3 Department of Health and Medical Sciences, University of Surrey, GUILDFORD, Surrey GU2 7TE, UK \\ ${ }^{4}$ Geisinger Holy Spirit, Camp Hill, PA, USA \\ 5 Telfer School of Management, University of Ottawa, Ottawa, Ontario, Canada, K1N 6N5 \\ ${ }^{6}$ Centre for Studies in Family Medicine, Department of Family Medicine, Interfaculty Program \\ in Public Health, Department of Epidemiology \& Biostatistics, Schulich School of Medicine \& \\ Dentistry, Western University, London, ON, Canada
}

\section{Summary}

Background: Patients' access to their computerised medical records (CMRs) is a legal right in many countries. However, little is reported about the benefit-risk associated with patients' online access to their CMRs.

Objective: To conduct a consensus exercise to assess the impact of patients' online access to their CMRs on the quality of care as defined in six domains by the Institute of Medicine (IOM), now the National Academy of Medicine (NAM).

Method: A five-round Delphi study was conducted. Round One explored experts' $(n=37)$ viewpoints on providing patients with access to their CMRs. Round Two rated the appropriateness of statements arising from Round One $(n=16)$. The third round was an online panel discussion of findings $(n=13)$ with the members of both the International Medical Informatics Association and the European Federation of Medical Informatics Primary Health Care Informatics Working Groups. Two additional rounds, a survey of the revised consensus statements and an online workshop, were carried out to further refine consensus statements.

Results: Thirty-seven responses from Round One were used as a basis to initially develop 15 statements which were categorised using loM's domains of care quality. The experts agreed that providing patients online access to their CMRs for bookings, results, and prescriptions increased efficiency and im- proved the quality of medical records. Experts also anticipated that patients would proactively use their online access to share data with different health care providers, including emergencies. However, experts differed on whether access to limited or summary data was more useful to patients than accessing their complete records. They thought online access would change recording practice, but they were unclear about the benefit-risk of high and onerous levels of security. The 5-round process, finally, produced 16 consensus statements. Conclusion: Patients' online access to their CMRs should be part of all CMR systems. It improves the process of health care, but further evidence is required about outcomes. Online access improves efficiency of bookings and other services. However, there is scope to improve many of the processes of care it purports to support, particularly the provision of a more effective interface and the protection of the vulnerable.

Keywords

Medical record systems, computerised; privacy; confidentiality; general practice; Delphi technique; patients' access to records

Yearb Med Inform 2018:156-62

http://dx.doi.org/10.1055/s-0038-1641202

\section{Introduction}

Patients' online access to their computerised medical records (CMRs) may benefit patients, but the evidence is less clear about outcomes [1]. A recent systematic review suggested the place of primary care CMRs is far from established in the emergency setting [2]. There are also concerns about equity of access to information and services across the "digital divide" between the less and more deprived members of the population [3]. CMR uptake is often limited by concerns about privacy [4-6]. Patients who have online access to their primary care CMR reports improved convenience and satisfaction [7, 8]. However, in the drive to implement online patients' access, concerns remain from clinicians about confidentiality, privacy, and patient safety [9-11]. Patients' online access to their CMRs can include doctors' visit notes and has the potential to improve the delivery of care $[12,13]$. Early studies have demonstrated the feasibility of online CMR access [13], including secure messaging with health care providers [14, 15], but clinicians have expressed concerns 
about patients' access causing confusion or worry to patients $[14,16]$. Patients' online access to their CMRs has also been reported to improve patient-clinician communication [17-19] and possibly may strengthen these relationships [11]. However, clinicians have concerns whether online access to CMRs may impact physician workload and threaten professional autonomy [20].

Patients' online access may improve patient safety by letting patients check their medication lists $[14,21]$ and correct discrepancies [16]. Online prescription renewal may improve medication adherence [14, 22], though there is reduced uptake from those with limited English proficiency [23]. Online access may improve the uptake of preventive care services, such as influenza immunisation and mammography for breast cancer screening [13, 24]. Online CMR access has also been provided for laboratory test results [18]; though concerns have been expressed about security [25], especially in case of abnormal test results $[1,26]$, and generally about communication protocols [24].

We conducted a Delphi study among informatics experts to explore the impact of patients' online access to their CMRs on the quality of care, with a focus on outcomes. We used the Institute of Medicine's (IoM), now the National Academy of Medicine (NAM), definition of the quality of care because of its focus on outcomes: "The degree to which health care services for individuals and populations increase the likelihood of desired health outcomes and are consistent with current professional knowledge".

\section{Methods}

\section{Consensus Exercise}

We recruited volunteer health informatics experts involved in the Primary Health Care Informatics Working Groups of the European Federation of Medical Informatics (EFMI) and the International Medical Informatics Association (IMIA) to conduct a three-round Delphi study, which we subsequently extended to five.

a. Round 1: Identifying the global perspectives of confidentiality, privacy, and security challenges associated with providing patients'access to their CMRs - an online survey

Round 1 was an online survey which aimed to explore health informatics experts' experiences with providing patients online access to their CMRs. We also inquired whether experts perceived challenges regarding confidentiality, privacy, or security to influence the provision of patients' access to their medical records. Finally, we inquired whether they thought providing patients with access to their CMRs could improve the process or care and health outcomes.

We used the responses from Round 1 to develop consensus statements for Round 2 .

\section{b. Round 2: Rating statements using the} RAND/UCLA appropriateness methodan online survey

We analysed the responses from Round 1 comparing them against the IoM domains of quality [27] (Table 1). The statements provided for Round 1 were divided into enablers and inhibitors of each of the six domains. We then consolidated all the enablers for each quality domain and integrated them into key statements. We repeated the process for inhibitors. We sent the statements as an online survey to the 37 experts who responded to Round 1 . Sixteen (43\%) responded to the survey. The list of statements is given in Table 2. We replaced the standard terms used in the UCLA/RAND appropriateness method, "Highly appropriate" and "Highly inappropriate", with "Strongly agree" and "Strongly disagree".

c. Round 3: Discussion of the findings by health informatics experts - an online panel discussion

The next round of the consensus process was an online panel discussion. Three separate online meetings were organised to engage panel members in different time zones. Thirteen experts (35\%) participated in this round.

Table 1 Number of final consensus statements for each of the Institute of Medicine domains of health care quality.

\begin{tabular}{|l|l|c|}
\hline Domain & \multicolumn{1}{|c|}{ Description } & $\begin{array}{c}\text { Consensus } \\
\text { statements }\end{array}$ \\
\hline Safe & $\begin{array}{l}\text { Avoiding harm to patients from the care that is intended to help } \\
\text { them. }\end{array}$ & 3 \\
\hline Effective & $\begin{array}{l}\text { Providing services based on scientific knowledge to all who } \\
\text { could benefit and refraining from providing services to those not } \\
\text { likely to benefit (avoiding underuse and misuse, respectively). }\end{array}$ & 3 \\
\hline $\begin{array}{l}\text { Patient- } \\
\text { centred }\end{array}$ & $\begin{array}{l}\text { Providing care that is respectful of and responsive to individual } \\
\text { patient preferences, needs, and values and ensuring that } \\
\text { patient values guide all clinical decisions. }\end{array}$ & \\
\hline Timely & $\begin{array}{l}\text { Reducing waits and sometimes harmful delays for both those } \\
\text { who receive and those who give care. }\end{array}$ & 2 \\
\hline Efficient & $\begin{array}{l}\text { Avoiding waste, including waste of equipment, supplies, ideas, } \\
\text { and energy. }\end{array}$ & 2 \\
\hline Equitable & $\begin{array}{l}\text { Providing care that does not vary in quality because of personal } \\
\text { characteristics such as gender, ethnicity, geographic location, } \\
\text { and socioeconomic status. }\end{array}$ & 2 \\
\hline
\end{tabular}


Table 2 Consensus statements generated from the analysis of Round l's responses (with Agreement written in green, Equivocation in brown, and Disagreement in red).

\section{SAFE}

Statement 1 - Allowing patients' access to their record increases the quality of CMRs by enabling patients validate (or correct errors) in their data. [Enabler] [Agreement] Statement 2 - Allowing patients' access to their record increases the security of CMRs by enabling patients identify potential improper access. [Enabler] [Disagreement]

Statement 3 - Making patient data available through online services introduces the risk of sensitive information being unnecessarily exposed to persons related to the patient (i.e. family members, friends). [Inhibitor] [Disagreement]

Statement 4 - Computing devices and infrastructure used to access online services would not provide a high level of security (e.g. password saving on browser or access to data through public computers/networks). [Inhibitor] [Disagreement]

\section{EFFECTIVE}

Statement 5 - Using online patient services such as appointment bookings or medication requests results in the effective utilisation of care provider resources. [Enabler] [Agreement] Statement 6 - Excessive patient data will reduce the effectiveness of patients' online experience. [Inhibitor] [Equivocation]

\section{PATIENT-CENTRED}

Statement 7 - Online access to patient services promotes patient independence and transparency. [Enabler] [Agreement]

Statement 8 - Online access to patient services enhances shared decision-making. [Enabler] [Disagreement]

Statement 9 - Access to patient data such as radiology results or lab results will not be cost-beneficial as it will not be used by the wider patient population. [Inhibitor] [Disagreement]

\section{TIMELY}

Statement 10 - Online patients' access to data is important in emergency situations where information systems of care providers are not integrated. [Enabler] [Agreement]

Statement 11 - Irrelevant data provided through online patient services may affect the data quality of CMRs. [Inhibitor] [Disagreement]

\section{EFFICIENT}

Statement 12 - Online patient services improve the efficiency of communication and data exchange between patients and care providers, resulting in the reduction of the waste of resources. [Enabler] [Agreement]

Statement 13 - Clinicians will have to improve the way medical notes are recorded to allow patients understand their CMR. [Inhibitor] [Disagreement]

\section{EQUITABLE}

Statement 14 - The equitable access to data provided by online patient services outweighs any risk of data privacy. [Enabler] [Equivocation]

Statement 15 - Privacy regulations have a negative impact on the equitable access to online patient services. [Inhibitor] [Disagreement]

\section{d. Round 4: Discussion of the revised con-} sensus statements by health informatics experts - an online panel discussion

Based on the feedback received in peer reviews, we revised the statements and conducted two online meetings to discuss the changes. Eleven experts (31\%) participated in this round.

\section{Results}

The process involved consulting an international panel of 37 experts from 10 countries: Australia, Canada, Croatia, Finland, New Zealand, Portugal, Spain, United Kingdom, USA, and Vietnam (Figure 1).

There was a good degree of consensus, as defined by the RAND/UCLA method [28] by the end of the final round (see Table 3 ). The statements for Round 3 (Table 2) had agreement on only a third $(5 / 15)$ of the statements; this had risen to three-quarters $(12 / 16)$ by the end the final round (Table 4). There were two principal reasons for this: firstly, the experts were considering the statements in the context of vulnerable groups; and secondly some of the early statements included a reason or mechanism. The respondents might have agreed with the statements but not the mechanism. We had an additional statement in the final round as the experts suggested to split one of the statements used in Round 3 (i.e. statement 7).

The statements and key findings related to the six domains of quality are set out below:

Safe: Panel experts agreed that better security measures would improve patient confidence. However, there was a range of views (equivocation) about identifying prescribing errors. This was due to the uncertainty whether patients have the necessary training to identify errors. Some experts felt that the accuracy of prescribing should principally be the clinician's responsibility. Our experts recognised there were concerns about privacy in the clinical community and uncertainty how to mitigate risk. There was such uncertainty about those who might be vulnerable, because of age or infirmity, or lack of competence that we inserted a stem to our statements, stating that they only applied to an adult competent to make decisions about his or her own care.

Effective: In Statements 4, 5, and 6, informatics experts generally saw services being more effective when patients had online access. However, there was a debate as to whether a summary access or a comprehensive access to CMRs was better. Whilst accepting that patients had a right to see any data recorded in their CMRs, a better interface and mechanism for navigating 


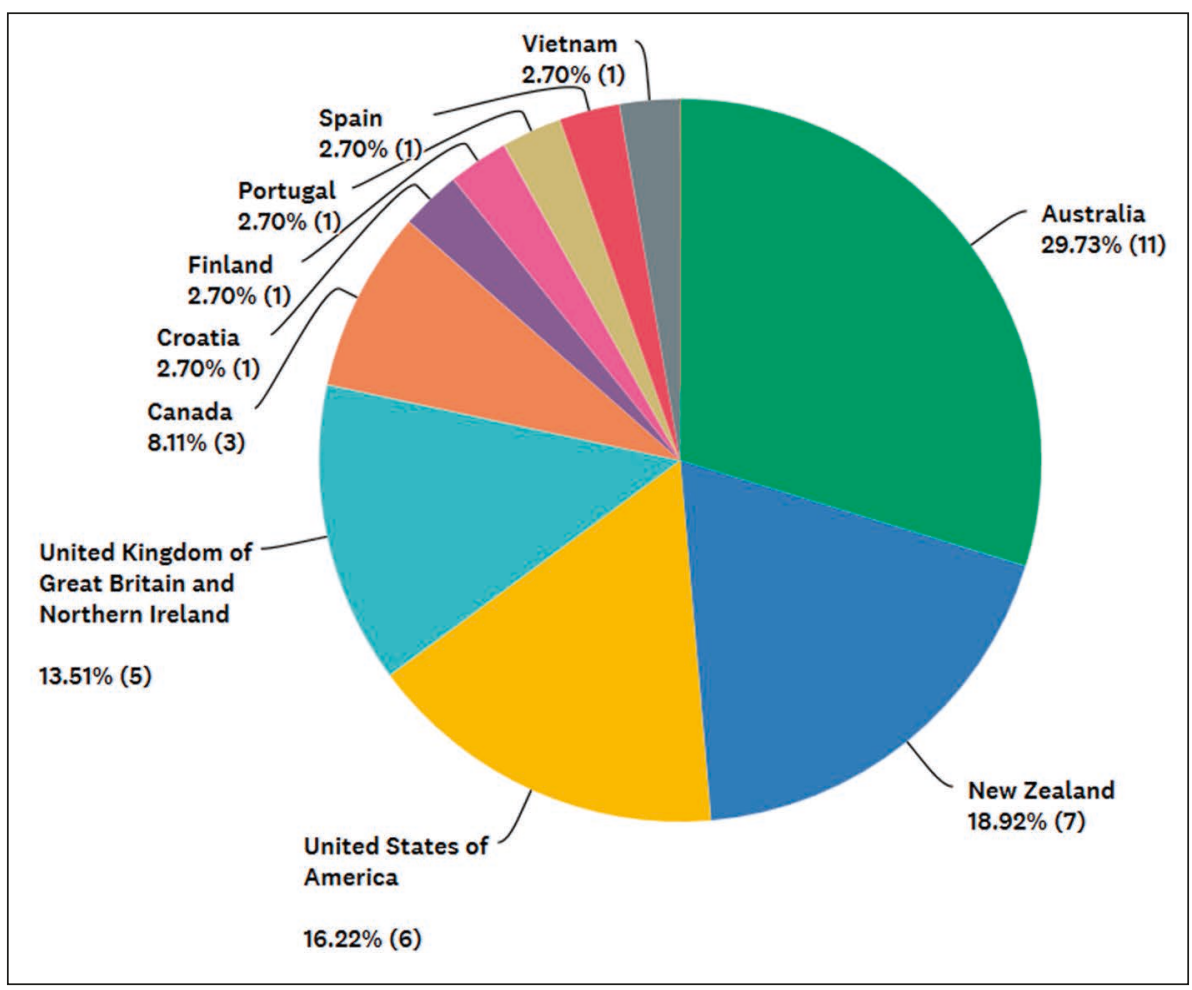

Fig. 1 Distribution of the health informatics experts who participated to the Round 1

Table 3 Summary of consensus levels achieved during surveys to assess the appropriateness of the statements by the experts' panel

\begin{tabular}{l|c|c|}
\hline & Round 3 (15 original statements) & Round 5 (16 revised statements) \\
\hline Agreement & 5 & 12 \\
Equivocation & 8 & 2 \\
Disagreement & 2 & 2 \\
\hline
\end{tabular}

CMRs were thought to be needed. The use of the word "excessive" in earlier rounds was thought to be unhelpful and removed.

Patient-centred: Informatics experts had strong agreement with all statements in this domain. Patient autonomy is a prerequisite for shared decision-making and relies on the availability of information. Access should promote independence and shared decision-making.

Timely: There was strong agreement on Statement 11 about the utility of CMR access during unplanned care. There was equivocation about Statement 12 that the data added by patients risk being a distraction, with panel members citing large numbers of observations presented as a "data dump" being hard to interpret.

Efficient: There was a strong agreement on Statement 13 that online services improve overall efficiency of CMRs. Experts agreed that CMRs are only efficient if the patient uses it and provided there is interoperability between health providers. The majority of participants believed that clinicians would have to improve the way medical notes are recorded to allow patients to understand their CMRs (Statement 14). Some participants reported positive feedback from "open notes", a project to share clinic notes with patients [29].

Equitable: Participants disagreed on statements related to equitable access (Statement 15 and Statement 16). Several panel members commented that access and privacy are neither linked nor inversely related. There was concern that online access may further widen disparities in health care, and the principal issue was the protection of the vulnerable.

\section{Discussion}

\section{Principal Findings}

Online booking of appointments, accessing results, requesting prescriptions, and other services are beneficial. CMR access may also promote autonomy, activation, and greater self-management. There was equivocation over whether patients should have comprehensive access to their CMRs or access to selected or summary data and if it was reasonable for patients to have a role in prescribing safety.

Whilst there was an agreement that online access improves many aspects of the process of care, there remained equivocation and disagreement about some of them; principally because the case has not been made that online access improves health outcomes.

The issues about the process of care were how to integrate the tasks arising out of patients' use of CMRs into the clinical workflow. Our experts were unclear whether it was right to weigh up the benefits of patients' access to their CMRs against the risk of security and privacy breaches. The level of risk was unclear to clinicians, and especially their responsibilities with respect to potentially vulnerable people including minors, older people, or competent adults who might be vulnerable to abuse.

\section{Implication of Findings}

Transactional processes (appointment booking, prescriptions, some test results) should become part of all CMR systems. 
Organisations like OpenEHR (http://www. openehr.org/) and informatics bodies could promote standard approaches that would facilitate the implementation of CMR systems [30].

Generally, online access improves the process of care. However, better interfaces with filters of data are needed for both patents and clinicians. Just providing access to a growing volume of data won't improve the process of care delivery. Safeguards are needed to ensure that online access would not deny vulnerable people confidential access to their clinician.

Online access has not yet been shown to improve the outcomes of care. We lack a benefit-risk assessment measure such as that which is used to assess the benefit and risk of a treatment [31]. Online access to CMRs will gradually normalise, as part of the online conversation we will increasingly have with our patients [32].

\section{Limitations of the Method}

We used an opportunistic sample of health informatics experts drawn from International Primary Care Health Informatics Working Groups. The structure of consensus statements could have been improved (use of double negatives and agreement to a single causal link). However, discussions that occurred in rounds four and five helped clarify many of the ambiguities in the responses we received.

\section{Conclusions}

There is international consistency in the agreement with structural and process aspects and impacts of providing patients with online access to their CMRs. Disagreement appeared to be driven by international variations in health care systems, cultures, and participants' practical experience. Online access to CMRs should be part of all medical record systems. Generally, online access supports the process of care. However, there remain important areas of tension around the process of turning data into information, keeping
Table 4 Revised consensus statements used for the final round (with Agreement written in green, Equivocation in brown, and Disagreement in red).

\section{SAFE}

Stem: For competent adult patients, online access to their computerised medical records (CMRs) has the following consequences:

Statement 1 - Patients may identify prescribing errors.

Mechanism: Patients may identify errors in their prescriptions when they have time to consider them in detail. [Enabler] Equivocation (Weighted average: 6.75)

Statement 2 - Confidential information may be improperly accessed by persons related to the patient.

Mechanism: Fear or actual breaches in confidentiality may inhibit patient disclosure. Pressures from family members, friends, carers, and others, which the patient may prefer not to happen, might occur. [Inhibitor] Weak Agreement (Weighted average: 6.04)

Statement 3 - Multi-factor authentication would improve patients' confidence in the security of their CMR data.

Mechanism: Methods similar to what is used in the banking industry (such as the use of physical security tokens, mobile phone apps, or digital authentication). [Enabler] Strong Agreement (Weighted average: 7.39)

\section{EFFECTIVE}

Stem: For competent adult patients, online access to their computerised medical records (CMRs) has the following consequences:

Statement 4 - Improve the quality of patients' CMR used for their care.

Mechanism: Enablers of this may include enabling patients to validate (or correct errors in) their data; understand more about their condition, and better frame questions at their next visit. [Enabler] Strong agreement (Weighted average: 7.3)

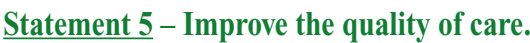

Mechanism: Many mechanisms may account for this, some are listed above. However, this gives the opportunity for experts to report if they overall feel that CMRs improve the quality of care. [Enabler] Weak agreement (Weighted average: 6.83)

Statement 6 - Reduce health care costs.

Mechanism: Enablers of this may include reducing the duplication of tests and improving slow sharing or missing information exchange between different health care providers. [Enabler] Weak agreement (Weighted average: 7.13)

\section{PATIENT-CENTRED}

Stem: For competent adult patients, online access to their computerised medical records (CMRs) has the following consequences:

Statement 7 - Promote self-management.

Mechanism: This may include patients accessing their CMRs to check previous medical results, which may help them set targets for their health until their next check-up and change their self-management behaviours. [Enabler] Strong agreement (Weighted average: 7.3)

$\underline{\text { Statement } 8}$ - Promote transparency in the patient-clinician relationship.

Mechanism: This may promote new ways of communication between patients and clinicians: patients can check clinicians' notes after their consultation. [Enabler] Strong agreement (Weighted average: 7.78)

Statement 9 - Promote shared decision-making. Mechanism: For example, patient using results which were provided by secondary care to discuss an issue with their primary care clinician. [Enabler] Strong agreement (Weighted average: 7.43 )

Statement 10 - Reduce patient costs.

Mechanism: Reduce patient costs related to consultation, such as travel expenses, patient contribution expenses, etc. [Enabler] Weak agreement (Weighted average: 6.7) 


\section{TIMELY}

\section{Stem: For competent adult patients, online access to their computerised medical records (CMRs) has the following consequences:}

Statement 11 - Facilitate health care in situations where unplanned care is needed and information systems of health care providers are not integrated.

Mechanism: Many mechanisms can be involved, such as emergencies in a hospital setting or urgent prescriptions of drugs in primary care. [Enabler] Strong agreement (Weighted average: 7.39 )

$\underline{\text { Statement } 12}$ - Data (text or other media) added by patients may distract more than assist their medical care.

Mechanism: Patients might add a large volume of data on an aspect of their care that is not the focus and which may overload their health care providers. This might include pictures, videos, or other data. [Inhibitor] Equivocation (Weighted average: 6.48)

\section{EFFICIENT}

Stem: For competent adult patients, online access to their computerised medical records (CMRs) has the following consequences:

$\underline{\text { Statement } 13}$ - Facilitate booking appointments and other transactions currently done face-to-face.

Mechanism: Enablers of this could be online self-services, such as appointment bookings or medication requests. [Enabler] Strong agreement (Weighted average: 8.17)

$\underline{\text { Statement } 14}$ - Elicit changes in clinicians' style of recording observational data in health records.

Mechanism: Clinicians will need to use accessible and accurate language so that patients understand their notes. [Enabler] Weak agreement (Weighted average: 6.87)

\section{EQUITABLE}

Stem: For competent adult patients, online access to their computerised medical records (CMRs) has the following consequences:

Statement 15 - Provide health benefits that outweigh any risk.

Mechanism: Online access could pose risks to privacy, security, confidentiality. [Enabler] Disagreement (Weighted average: 6.67)

$\underline{\text { Statement } 16}$ - Privacy regulations have a negative impact on the online access to patient information.

Mechanism: The problems for patients either to set-up an account and/or to login and/ or to manage authentication may dissuade them from using the online system. [Inhibitor] Disagreement (Weighted average: 5.73)

provider workload manageable, and how to protect vulnerable people. Whilst it remains unclear whether online access results in better outcomes, this should not be used as a reason to stop their increasing use. Our focus should be on overcoming the challenges of integrating online access into the process of care.

\section{Acknowledgements}

We would like to acknowledge the contributions of the Primary Health Care Informatics Working Groups of EFMI and IMIA to the study. This paper describes the work of volunteers.

\section{References}

1. Shah SG, Fitton R, Hannan A, Fisher,B, Young $\mathrm{T}$, Barnett,J. Accessing personal medical records online: a means to what ends? Int J Med Inform 2015;84(2):111-8.

2. Bowden T, Coiera E. The role and benefits of accessing primary care patient records during unscheduled care: a systematic review. BMC Med Inform Decis Mak 2017;17(1):138.

3. Adler-Milstein J, Holmgren AJ, Kralovec P, Worzala C, Searcy T, Patel V. Electronic health record adoption in US hospitals: the emergence of a digital "advanced use" divide. J Am Med Inform Assoc 2017;24(6):1142-8.

4. Baker MTM, Mawby R. The Future of GP Out-ofHours Care. An RCGP Position Statement. London, UK: Royal College of General Practitioners;
2014. Available from: http://www.rcgp.org.uk/-/ media/Files/Policy/A-Z-policy/RCGP-The-Future-of-GP-Out-of-Hours-Care-2015.ashx?la=en.

5. Practitioners RCoG. A Vision for General Practice in the future NHS: The 2022 GP. London, UK: Royal College of General Practitioners; 2013. Available from: http://www.rcgp.org.uk/ campaign-home/ /media/files/policy/a-z-policy/ the-2022-gp-a-vision-for-general-practice-in-thefuture-nhs.ashx.

6. Ware JMR. Patient access to general practice: ideas and challenges from the front line. London, UK: Royal College of General Practitioners; 2015. Available from: https://www.hiirc.org. nz/page/55381/patient-access-to-general-practice-ideas/;jsessionid=A570B42B376B6E92FBFAEB04FABD2C04? content Type $=251 \&$ sec tion $=13825$.

7. Mold F, de Lusignan S, Sheikh A, Majeed A, Wyatt JC, Quinn T, et al. Patients' online access to their electronic health records and linked online services: a systematic review in primary care. $\mathrm{Br} \mathrm{J}$ Gen Pract 2015;65(632):e141-51.

8. de Lusignan S, Mold F, Sheikh A, Majeed A, Wyatt JC, Quinn T, et al. Patients' online access to their electronic health records and linked online services: a systematic interpretative review. BMJ Open 2014;4(9):e006021.

9. Iacobucci G. Patients promised online access to their medical records by 2018. BMJ (Clinical research ed) 2015;351:h4740.

10. Woodman J, Sohal AH, Gilbert R, Feder G. Online access to medical records: finding ways to minimise harms. Br J Gen Pract 2015;65(635):280-1.

11. Vodicka E, Mejilla R, Leveille SG, Ralston JD, Darer JD, Delbanco,T, et al. Online access to doctors' notes: patient concerns about privacy. J Med Internet Res 2013;15(9):e208.

12. Wade-Vuturo AE, Mayberry LS, Osborn CY. Secure messaging and diabetes management: experiences and perspectives of patient portal users. J Am Med Inform Assoc 2013;20(3):519-25.

13. Hannan A. Providing patients online access to their primary care computerised medical records: a case study of sharing and caring. Inform Prim Care 2010;18(1):41-9.

14. Nagykaldi Z, Aspy CB, Chou A, Mold JW. Impact of a Wellness Portal on the delivery of patient-centered preventive care. J Am Board Fam Med 2012;25(2):158-67.

15. Delbanco T, Walker J, Bell SK, Darer JD, Elmore $\mathrm{JG}$, Farag $\mathrm{N}$, et al. Inviting patients to read their doctors' notes: a quasi-experimental study and a look ahead. Ann Intern Med 2012;157(7):461-70.

16. Root J, Oster NV, Jackson SL, Mejilla R, Walker J, Elmore JG. Characteristics of Patients Who Report Confusion After Reading Their Primary Care Clinic Notes Online. Health Commun 2016;31(6):778-81.

17. Kummervold PE, Trondsen M, Andreassen H, Gammon D, Hjortdahl P. [Patient-physician interaction over the internet]. Tidsskrift for den Norske laegeforening : tidsskrift for praktisk medicin, ny raekke. 2004;124(20):2633-6.

18. Lin CT, Wittevrongel L, Moore L, Beaty BL, Ross SE. An Internet-based patient-provider communi- 
cation system: randomized controlled trial. J Med Internet Res 2005;7(4):e47.

19. Matheny ME, Gandhi TK, Orav EJ, Ladak-Merchant Z, Bates DW, Kuperman GJ, et al. Impact of an automated test results management system on patients' satisfaction about test result communication. Arch Intern Med 2007;167(20):2233-9.

20. Alander T, Scandurra I. Experiences of Healthcare Professionals to the Introduction in Sweden of a Public eHealth Service: Patients' Online Access to their Electronic Health Records. Stud Health Technol Inform 2015;216:153-7.

21. Schnipper JL, Gandhi TK, Wald JS, Grant RW, Poon EG, Volk LA, et al. Effects of an online personal health record on medication accuracy and safety: a cluster-randomized trial. J Am Med Inform Assoc 2012;19(5):728-34.

22. Sarkar U, Lyles CR, Parker MM, Allen J, Nguyen $\mathrm{R}$, Moffet $\mathrm{HH}$, et al. Use of the refill function through an online patient portal is associated with improved adherence to statins in an integrated health system. Med Care 2014;52(3):194-201.

23. Moreno G, Lin EH, Chang E, Johnson RL, Berthoud H, Solomon CC, et al. Disparities in the Use of Internet and Telephone Medication Refills among Linguistically Diverse Patients. J Gen Inter
Med 2016;31(3):282-8.

24. Wright A, Poon EG, Wald J, Feblowitz J, Pang JE, Schnipper JL, et al. Randomized controlled trial of health maintenance reminders provided directly to patients through an electronic PHR. J Gen Intern Med 2012;27(1):85-92.

25. Litchfield IJ, Bentham LM, Lilford RJ, Greenfield SM. Test result communication in primary care: clinical and office staff perspectives. Fam Pract 2014;31(5):592-7.

26. Mak G, Smith Fowler H, Leaver C, Hagens S, Zelmer J. The Effects of Web-Based Patient Access to Laboratory Results in British Columbia: A Patient Survey on Comprehension and Anxiety. J Med Internet Res 2015;17(8):e191.

27. Institute of Medicine Committee on Quality of Health Care in America. Crossing the Quality Chasm: A New Health System for the $21^{\text {st }}$ Century. Washington (DC): National Academies Press (US); 2001 .

28. Fitch KBSJ, Aguilar MD, Burnand B, LaCalle JR, Lazaro P, van het Loo M, et al. The RAND/UCLA Appropriateness Method User's Manual; 2001.

29. Delbanco T, Walker J, Darer JD, Elmore JG, Feldman HJ, Leveille SG, et al. Open notes: doctors and patients signing on. Ann Intern Med
2010;153(2):121-5.

30. Buck J, Garde S, Kohl CD, Knaup-Gregori P. Towards a comprehensive electronic patient record to support an innovative individual care concept for premature infants using the openEHR approach. Int J Med Inform 2009;78(8):521-31.

31. Ara R, Wailoo A. NICE Decision Support Unit Technical Support Documents. NICE DSU Technical Support Document 12: The Use of Health State Utility Values in Decision Models. London: National Institute for Health and Care Excellence (NICE)unless otherwise stated; 2011.

32. Weiner JP. Doctor-patient communication in the e-health era. Isr J Health Policy Res 2012;1(1):33.

Correspondence to:
Harshana Liyanage
Department of Clinical \& Experimental Medicine
University of Surrey
GUILDFORD
Surrey GU2 7XH
United Kingdom
E-mail: h.s.liyanage@surrey.ac.uk

